\title{
Le nazisme, un régime de la citation
}

\section{Eric Michaud}

\section{(2) OpenEdition}

\section{Journals}

Édition électronique

URL : http://journals.openedition.org/imagesrevues/885

DOI : 10.4000/imagesrevues.885

ISSN : 1778-3801

\section{Éditeur :}

Centre d'Histoire et Théorie des Arts, Groupe d'Anthropologie Historique de l'Occident Médiéval, Laboratoire d'Anthropologie Sociale, UMR 8210 Anthropologie et Histoire des Mondes Antiques

\section{Référence électronique}

Eric Michaud, «Le nazisme, un régime de la citation », Images Re-vues [En ligne], Hors-série 1 | 2008, mis en ligne le 21 avril 2011, consulté le 30 janvier 2021. URL : http://journals.openedition.org/ imagesrevues/885; DOI : https://doi.org/10.4000/imagesrevues.885

Ce document a été généré automatiquement le 30 janvier 2021.

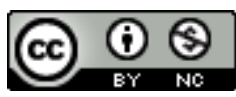

Images Re-vues est mise à disposition selon les termes de la Licence Creative Commons Attribution Pas d'Utilisation Commerciale 4.0 International. 


\title{
Le nazisme, un régime de la citation
}

\author{
Eric Michaud
}

1 C'est à Walter Benjamin que l'on attribue généralement cette belle idée que l'histoire est toujours écrite par les vainqueurs. Mais ainsi formulée, cette attribution mérite cependant quelques éclaircissements. Lorsque, dans ses fameuses thèses sur la philosophie de l'histoire écrites durant les premiers mois de l'année 1940, Benjamin affirmait en

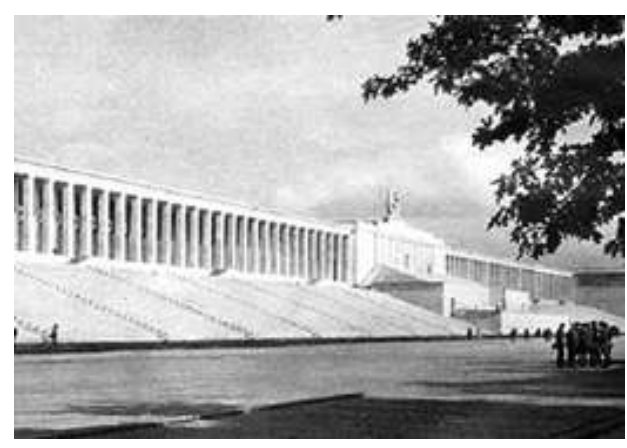
substance que le travail de l'historiciste, toujours en empathie avec les vainqueurs du passé, bénéficiait inévitablement aux maîtres du moment, c'était pour opposer à cette histoire des vainqueurs celle de l'historien matérialiste dont il définissait la tâche comme étant de «brosser l'histoire à rebrousse-poil ", c'est-à-dire sans empathie ${ }^{1}$ : ainsi l'historien matérialiste devait-il se tenir à l'écart du mouvement de transmission des biens culturels - ce butin que les vainqueurs, toujours, portent dans leur cortège triomphal. S'il affirmait que le matérialisme historique avait rompu avec «la méthode de l'empathie ", lui-même préconisait pourtant une certaine empathie - mais c'était avec les vaincus du passé et non avec les vainqueurs; c'était avec «la tradition des opprimés ", pour tenter de réactiver les possibilités non advenues que recélait l'histoire. Ce faisant, Benjamin se situait certainement dans la perspective d'une victoire future - mais d'une victoire remportée cette fois sur toute opposition entre vainqueurs et vaincus, une victoire enfin en tous points comparable à celle que promettait la venue d'un temps messianique: "Ce n'est qu'à l'humanité rédimée qu'échoit pleinement son passé. C'est dire que pour elle seule son passé est devenu intégralement citable. Chacun des instants qu'elle a vécus devient 'une citation à l'ordre du jour' - et ce jour est justement celui du Jugement dernier ${ }^{2}$ ».

2 Or s'il opposait ainsi, aux citations arrachées aux vaincus pour composer le butin des vainqueurs, le concept d'un passé devenu intégralement citable, c'était parce que l'idée selon laquelle il n'y a d'histoire qu'écrite par les vainqueurs circulait en Allemagne 
depuis plusieurs années déjà - mais c'était sous la plume des penseurs de la droite et de l'extrême droite qu'on la trouvait alors. A l'évidence, c'était à ceux-ci qu'il répondait d'abord en stigmatisant la citation empathique des vainqueurs.

Une telle idée s'énonçait en effet, en des termes à la fois proches et distants de ceux de Benjamin, dans Le Travailleur que publiait Ernst Jünger en 1932; ou bien encore de façon plus diffuse chez le Heidegger de Sein und Zeit, en 1927, lorsque ce dernier évoquait la nécessité, pour qui veut surmonter la médiocrité de la vie quotidienne qui domine la masse, de « choisir ses héros » parmi ceux qui ont peuplé l'histoire, afin de se rendre «libre pour la poursuite du combat et pour la fidélité au répétable ${ }^{3}$ ». Ainsi entendue, la répétition, on le sait, n'était pas pour Heidegger restitution du passé, mais décision d'assumer une transmission, un héritage, pour affirmer le destin historial et authentique d'un peuple.

4 La formulation d'Ernst Jünger, cinq ans plus tard, était bien plus forte et bien plus radicale aussi. Elle apparaissait d'abord au détour d'une page où, à la veille de l'effondrement de la République de Weimar, évoquant la puissante «Figure du Travailleur » dont la " Domination » entraînerait « nécessairement des solutions d'une ampleur planétaire et impérialiste", Jünger soulignait combien cette domination impliquerait non seulement une "administration de l'espace, mais en outre une administration du temps». Et cette administration du temps, précisait-il encore, consistera en « un complet renversement de la vision de l'histoire, de l'appréciation et de l'administration des performances historiques». Pourquoi un tel renversement? Parce que «le vainqueur écrit l'histoire et détermine son arbre généalogique ${ }^{4}$ ». Et soulignant plus loin que dans ce renversement « l'art n'est pas moyen mais objet de la mutation », Jünger ajoutait encore : « De même que le vainqueur écrit l'histoire, c'est-àdire se crée son propre mythe, de même il détermine ce qui doit avoir valeur d'art $"^{5}$.

Il reviendrait donc au vainqueur non seulement de redessiner l'espace et de réécrire l'histoire, mais aussi de décider en matière d'art entre ce qui vaut et ce qui ne vaut plus. Pourquoi donc une si haute importance conférée à l'art ? C'est que face à la Figure du Travailleur "sur le point de faire son entrée dans l'histoire", les ordres anciens s'effondrent pour laisser place à la "construction organique». A l'activité muséale, "dernière oasis de la sécurité bourgeoise", s'oppose le nouveau "paysage de chantier » où se révèle « l'identité de l'art et d'une suprême force de vie emplissant la totalité de l'espace ${ }^{6} »$. Dans ce renversement sans précédent, la figure du vaincu, recouverte par l'écriture du nouveau "mythe ", s'effacera de l'histoire en même temps que lui sera retiré tout jugement de valeur esthétique. Et cette figure, disait Jünger, est celle du «bourgeois », totalement dépourvue d'un sentiment pourtant si nécessaire de supériorité, sans conscience d'aucune originalité, sans assurance de jugement. Voilà, concluait-il, pourquoi le bourgeois "succombe sans défense et sans position personnelle au démonisme de tout phénomène historique, raison pour laquelle il tend à conférer puissance sur lui-même à toute grandeur historique qu'il est en train de considérer. De là vient aussi qu'on peut le battre à l'aide de n'importe quelle citation ${ }^{7}$.

6 Abandonnons à Jünger sa conception, si proche de celle du nazisme, du règne de la «Domination du Travailleur» - cette société sans classe dont il désirait la venue; retenons seulement son assertion que l'on peut battre le bourgeois avec n'importe quelle citation, puisque l'exhibition de toute « grandeur historique » est propre à le soumettre. Là réside sans aucun doute l'un des secrets de la fascination d'abord paralysante qu'a exercée le national-socialisme sur le peuple allemand. Car si les nazis, une fois arrivés 
au pouvoir, ont pu comme l'avait annoncé Jünger réécrire l'histoire et déterminer leur arbre généalogique, s'ils ont pu créer leur propre mythe et déterminer ce qui aurait désormais valeur d'art, ce fut en déversant sur l'Allemagne et les Allemands une avalanche de citations, tant verbales que visuelles. Des citations qui devenaient entre leurs mains des puissances d'autant plus contraignantes qu'elles précédaient, accompagnaient et prolongeaient l'usage de la force physique. Elles remplissaient ainsi exactement la fonction que Hitler, dans Mein Kampf, avait assigné à la propagande qu'il définissait comme " une Weltanschauung appuyée par la terreur ${ }^{8}$ ». Et cette profusion de citations verbales et plastiques envahissant tous les espaces publics, plongeant douze ans durant les Allemands dans l'atmosphère de chantier qu'avait exaltée Jünger, les immergeant dans un paysage de "mobilisation totale", allait bientôt leur fabriquer une mémoire nouvelle et singulière.

7 L'usage de la citation, cette modalité devenue ordinaire de l'invocation d'une auctoritas pour légitimer le discours de celui qui l'énonce, est comme on sait d'invention relativement récente. Ainsi Chateaubriand, dans la Préface de 1831 à ses Etudes historiques, remarquait-il que les Anciens, concevant «l'histoire autrement que nous ", la regardaient "comme un simple enseignement " . De ce fait, ils "attachaient peu d'importance à la vérité matérielle ; pourvu qu'il y eût un fait vrai ou faux à raconter, que ce fait offrit un grand spectacle ou une leçon de morale et de politique, cela leur suffisait. Délivrés de ces immenses lectures sous lesquelles l'imagination et la mémoire sont également écrasées, ils avaient peu de documents à consulter; leurs citations ne sont presque rien, et quand ils renvoient à une autorité, c'est presque toujours sans indication précise.» Ainsi, ajoutait-il, « deux citations vagues dans Hérodote, pas une dans Thucydide, deux ou trois dans Tite-Live, et treize dans Tacite, forment tout le corps des autorités de ces historiens ». Mais les temps avaient changé : maintenant que «l'histoire est une encyclopédie », se plaignait Chateaubriand, » il y faut tout faire entrer ».

Lorsqu'il entreprend de retracer sa propre histoire en la confondant avec celles de l'Allemagne récente et des commencements du nazisme, Adolf Hitler se situe incontestablement dans la perspective de l'Historia magistra vitae des Grecs et des Romains dont il revendique l'héritage: on ne trouve dans Mein Kampf presqu'aucune citation. C'est à l'évidence l'enseignement de cette triple histoire qu'il prétend livrer aux Allemands, en attachant » peu d'importance à la vérité matérielle ». Si l'on excepte les guillemets qui font place à ce qu'il nomme "la voix de ma conscience», on ne relève, dans cet ouvrage de près de huit cents pages, que deux vers extraits sans référence du Wallenstein de Schiller ${ }^{10}$, un mot de Schopenhauer dont le nom reste tu également ${ }^{11}$, deux brèves sentences de Moltke et de Bismarck, une phrase sans aucune référence extraite du Serment du Rütli de Guillaume Tell (« Nous sommes tous un peuple uni de frères $»^{12}$ ), un mot adapté de Frédéric le Grand, mais cité sans guillemets et sans nom d'auteur ${ }^{13}$, enfin deux ou trois " proverbes allemands ", plus ou moins adaptés eux aussi ${ }^{14}$. La visée de Hitler est simple : les rares citations tirées exclusivement d'auteurs allemands, noyées dans le récit de sa propre expérience, doivent avoir valeur d'exempla pour un peuple allemand qu'il faut ramener au sens de sa propre " race » et convaincre de la nécessité d'un Etat raciste-völkisch.

Or si le texte de Mein Kampf est cité en toute occasion et en tout lieu sous le Troisième Reich, excédant constamment les limites du livre pour imprégner la vie quotidienne tout entière et devenir l'autorité suprême de la Communauté du Peuple, ce fut 
précisément en raison de l'extrême rareté des autorités invoquées par un ouvrage qui devait remplir la fonction de nouvelle «bible» des Allemands - avec un tirage atteignant dix millions d'exemplaires en 1943.

D'un côté, en exposant la Weltanschauung raciste par simples assertions, en postulant la supériorité de la race aryenne au seul motif qu'elle serait la seule race "créatrice de culture ", Hitler n'a en effet nul besoin de fonder sa propre autorité : représentant la race supérieure dont il se pose tout à la fois en gardien et en guide, il en contient quasi naturellement et biologiquement toutes les autorités passées dont il est l'incarnation ultime. Cette autorité du Führer émanant de la seule appartenance raciale, le juriste Carl Schmitt lui donne sa légitimité dès les débuts du régime par le principe de l'Artgleichheit : c'est la « similitude de race » qui fonde la direction, la Führung politique de Hitler. Le concept de Führung, explique Schmitt, "est un concept d'actualité immédiate et de présence réelle [realer Präsenz]. C'est la raison pour laquelle il demande, comme exigence concrète, une inconditionnelle similitude de race entre le Führer et ceux qui le suivent [die Gefolgschaft]. C'est sur cette Artgleichheit que repose aussi bien le contact constant et infaillible entre le Führer et la Gefolgschaft que leur fidélité mutuelle. Seule cette Artgleichheit peut empêcher que la puissance du Führer ne devienne tyrannie et volonté arbitraire. (...) La similitude de race du peuple uni et d'accord en lui-même est aussi la plus incontournable [die unumgänglichste] condition et le fondement du concept de Führung politique ${ }^{15} »$. Il faut rappeler ici combien la langue du nazisme fait des mots Rasse (la race) et Art (l'espèce, mais aussi la manière d'être) de quasi synonymes. Ainsi Carl Schmitt peut-il ajouter : «Lorsque la pensée de la race [Rasse] fut sans cesse mise au coeur du Congrès des Juristes Allemands Nationalsocialistes de Leipzig en 1933, (...), ce ne fut pas comme un postulat de l'imagination théorique. Sans le principe de l'Artgleichheit, l'Etat national-socialiste ne pourrait exister et sa vie juridique ne serait pas pensable ${ }^{16}$.

11 Mais d'un autre côté, pour tous ceux qui citent cette nouvelle bible allemande prétendant fonder le mythe racial, il faut au contraire constamment confirmer, étayer, justifier et illustrer la Weltanschauung raciste au moyen d'un appareil considérable de citations prélevées sur le corps de la race, depuis ses origines et jusqu'à son actualité présente. Il faut sans relâche donner corps au mythe de la race supérieure afin qu'il devienne en effet l'objet d'une croyance commune. C'est ainsi qu'avec l'arrivée au pouvoir du national-socialisme, la citation, pour la première fois dans l'histoire sans doute, "fait système » en ce sens que rien, rigoureusement rien, ne saurait exister ni prendre corps sans elle. Chacune des citations dont usent et abusent les dirigeants et les idéologues constitue l'une des pièces d'un corps de doctrine qui a pour nom la Weltanschauung national-socialiste, et dont le caractère religieux est expressément formulé par Hitler, lorsqu'il la compare à «ce que les dogmes représentent pour la foi $»^{17}$.

12 Sans doute l'usage des citations pour réécrire l'histoire et légitimer ainsi certains choix politiques n'est-il, en lui-même, nullement propre au national-socialisme. "Toute sélection de matériel - fait justement observer Hannah Arendt - est en un sens une intervention dans l'histoire, et tous les critères de sélection placent le cours historique des événements dans certaines conditions dont l'homme est l'auteur ${ }^{18}$ ». Mais le racisme, vrai centre de la foi nouvelle autour duquel le nazisme va déployer la quasi totalité de ses citations visuelles et verbales, a pour conséquence logique un très singulier rapport à l'histoire : par ses critères de sélection, il ne cesse d'en user pour 
affirmer sa propre intemporalité, il s'y enracine en même temps qu'il la nie. Car le seul discours historique que reconnaisse et pratique le racisme est en effet le discours sur la race, c'est-à-dire - très littéralement - la généalogie. De sorte qu'avec la victoire du national-socialisme, toute citation de Maitre Ekhart, de Luther, de Kant ou de Wagner devient moins l'invocation d'un moment de l'histoire de la pensée allemande sous l'autorité de laquelle on se place, qu'une preuve de l'identité transhistorique de la substance raciale du peuple allemand, de l'« éternelle substance allemande». Le nazisme vainqueur répond ainsi à la double exigence de Jünger et de Heidegger : il "détermine son arbre généalogique » comme le voulait le premier, et il «choisit ses héros" afin de se rendre «libre pour la poursuite du combat et pour la fidélité au répétable ", comme l'avait formulé le second.

13 Aussi, lorsqu'un idéologue ou un dignitaire du Troisième Reich cite l'un des "Grands Allemands » du passé, c'est toujours le même sang qui coule, c'est toujours la même substance qui parle, c'est la race qui se parle à elle-même, consciente d'elle-même et totalement présente à elle-même. C'est le même sang qui circule dans un grand corps éternel, dont le Führer est comme le cœur qui pompe et irrigue ce corps pour en renouveler constamment les cellules. Ainsi le Volkskörper, le corps du peuple allemand n'a-t-il pas d'histoire : il s'actualise continûment dans un présent éternel, qui contient son passé autant que son avenir. Mais si ce corps n'a pas d'histoire, il a par contre une mémoire. Celle-ci se présente comme un vaste magasin où se trouvent, pêle-mêle, toutes les Leistungen, toutes les « réalisations » de la race depuis ses origines, c'est-àdire toutes les preuves de sa noble ascendance et de sa supériorité créatrice. Il faut imaginer ce magasin comme un entrepôt qui ne contiendrait que les produits d'une seule marque, ou bien encore comme le réceptacle des sécrétions d'un seul corps organique.

Sous le Troisième Reich, la pratique de la citation se présente donc comme une opération patrimoniale dont la fonction est de fabriquer une mémoire singulière au Volkskörper, ce corps du peuple allemand, soit d'obtenir qu'enfin une seule âme habitât un seul corps. Hitler, dans Mein Kampf, avait mis en garde les Allemands : « Les peuples qui renoncent à maintenir la pureté de leur race renoncent, du même coup, à l'unité de leur âme dans toutes ses manifestations ${ }^{19}$ ». Et cette unité de l'âme exige donc la sélection des manifestations passées du seul génie de la race, puisque de la pureté de la mémoire dépend aussi la pureté de la race.

Presque absentes de Mein Kampf, les citations verbales sont à l'inverse pléthoriques dans l'autre grand ouvrage de référence du régime: Le Mythe du 20e siècle, publié par Alfred Rosenberg en 1930, et dont le tirage atteindra bientôt le million d'exemplaires. Si Rosenberg passe pour avoir été l'un des grands «maîtres du Troisième Reich », c'est certainement parce qu'il est essentiellement un grand maître de la citation : c'est avec Luther, Schopenhauer, Wagner, Lagarde ou Meister Eckhart, qu'il construit pièce par pièce son Mythe du 20e siècle comme la "Renaissance " d'une pensée racialement déterminée, authentiquement "allemande» puisque élaborée par ces "Grands Allemands » dont la grandeur même des oeuvres atteste la supériorité raciale de ce peuple. Encore faut-il savoir séparer chaque fois le bon grain de l'ivraie, c'est-à-dire la part «masculine » de la part « féminine » de chacun de ces Grands Allemands : ainsi y a-t-il un «bon» et un «mauvais» Goethe, comme il y a un "bon» et un mauvais schopenhauer. 
16 Avec Faust, dit Rosenberg, Goethe a su représenter « l'essence » de la germanité - c'està-dire « l'éternel » - et devenir ainsi « le gardien et le protecteur de notre disposition »; mais il est nécessaire d'identifier le mauvais Goethe, celui qui « refusait la violence d'une idée façonnant un type ", celui qui « se refusait à reconnaître la dictature d'une pensée sans laquelle pourtant un peuple ne demeure jamais un peuple ${ }^{20}$ ». De même le Schopenhauer du Monde comme volonté et comme représentation est-il divisé entre ses deux natures contraires, qu'il tenta, mais sans succès, de réconcilier en identifiant la volonté à l'instinct aveugle - alors, commente Rosenberg, que c'est « la volonté (qui) maîtrise l'instinct et en triomphe ». Malgré cette belle certitude quant à la supériorité de la volonté, lorsqu'il en vient à citer Kant et son analyse du jugement de goût, c'est au nom de l'instinct qu'il la réduit aux dimensions de la communauté raciale: "L'universalité' du jugement de goût, assure-t-il, ne peut provenir que d'un idéal de beauté racial-völkisch et ne s'applique qu'à ceux qui, consciemment ou inconsciemment, portent dans leur coeur la même Idée de la beauté ${ }^{21}$ ". Comme l'écrit de son côté l'architecte et professeur Schultze-Naumburg, l'un des grands idéologue de l'art biologiquement déterminé : «L'artiste, qu'il le veuille ou non, ne peut pas échapper à son propre corps ${ }^{22} »$.

La sélection des citations sur des critères raciaux n'impose donc pas seulement d'exclure tout discours d'origine racialement impure : puisqu'au sein même de la race créatrice se perpétue l'éternel combat entre les principes de sa conservation et ceux de sa dissolution, il est proprement « vital » de ne sélectionner, chez les Grands Allemands eux-mêmes et leurs ancêtres aryens, que les propos capables de maintenir vivante et toujours identique à elle-même l'éternelle substance de la race.

18 Ainsi, les auctoritates des Grands Allemands ne sont-elles jamais convoquées pour seulement légitimer le racisme nazi, mais aussi et surtout pour le légitimer en tant qu'il constituerait l'essence éternelle de la race dont il est, du même coup, promu au rang de véritable gardien : le racisme est le gardien de la race comme le Volksgeist est le génie protecteur du Volkskörper, ou comme l'« Idée» nationale-socialiste garantit la conservation de la forme qui l'incarne. C'est cette circularité qui fait de toute citation prélevée sur le corps de la race une auto-citation, et de toute référence une autoréférence. Pour ce qui est du sens de cette circularité et de cette clôture absolue sur soimême, la palme revient sans doute à Rosenberg qui jalonne son Mythe du 20e siècle d'une citation de Maître Eckhart qui doit assigner au peuple allemand son but ultime : «Etre un avec soi-même. » De sorte que ce qui désignait chez Maître Eckhart l'union mystique de l'individu singulier avec son Dieu devient, chez Rosenberg, l'injonction d'une relation exclusive de la Communauté raciale avec son génie protecteur.

Mais ce n'est bien sûr que par leur diffusion et leur exposition dans les espaces publics que les citations, sous le Troisième Reich, atteignent leur véritable but qui est d'imprégner du sens de la race la vie quotidienne tout entière. C'est ainsi que peu avant le référendum sur l'Anschluss, une immense banderolle tendue à Vienne sur la façade de la Maison Adolf Loos interpelle le passant: «Gleiches Blut gehört in ein gemeinsames Reich! Un sang de même nature appartient à un Reich commun! Adolf Hitler ". C'est cependant au sein des entreprises et sur les lieux de travail que se joue, tout autant qu'au plan strictement biologique, l'appartenance au Reich commun. Car lorsque Hitler, dès septembre 1933, affirme qu'« on ne peut pas conclure de la race à la capacité, mais de la capacité à la race $^{23}$ ", il signifie bien qu'au sang doit s'ajouter le 
« travail créateur » qui devra donner consistance au mythe, dessinant les contours de la race par ses performances et ses réalisations.

D'innombrables sentences s'étalant sur les murs des entreprises allemandes répondent ainsi au exigences formulées en 1938 par le très remarquable guide intitulé Das Taschenbuch Schönheit der Arbeit. Ce "petit livre de la Beauté du Travail », rédigé par Anatol von Hübbenet, préfacé par Albert Speer, chef du Bureau de la Beauté du Travail depuis 1934, présente, autant à l'intention des dirigeants d'entreprise qu'à celle d'un plus vaste public, les réalisations de ce Bureau dont la mission est de rendre au travail la «noblesse » dont le christianisme et le «judéo-bolchévisme » l'avait privé en en faisant une "punition ». Consacré aux Wandsprüche ou "sentences murales », soit aux citations qui, renouvelées chaque semaine ou chaque mois, prennent place sur les murs des entreprises du Reich, l'un des chapitres de ce guide s'ouvre par un commentaire édifiant: "Les sentences des hommes exceptionnels de l'Histoire et du présent contraignent (zwingen) toujours et encore à la méditation et à faire retour sur soi-même (Selbstbesinnen). L'apposition de sentences sur les murs est devenue aujourd'hui une pratique générale. Ce n'est pas le fait du hasard, mais cela satisfait au goût qui s'est réveillé dans notre peuple pour la tradition et pour la vénération de nos grands hommes ${ }^{24} »$.

21 A la suite de recommandations techniques sur la façon de disposer, de manière décorative et percutante à la fois, les sentences les mieux appropriées à chacun des espaces des entreprises, quelques photographies illustrent la mise en œuvre de ces principes (Fig. 1). Sur le mur d'un atelier où trois mécaniciens s'affairent autour d'une automobile, un Wandspruch cite Adolf Hitler: "Nur wer dauernd nach Höchstleistungen strebt, kann sich in der Welt durchsetzen - Seul celui qui s'efforce durablement vers les plus hautes performances (Leistungen) peut se faire une place dans le monde. » Un autre Wandspruch, dans une salle de lecture, cite le Dr Goebbels : «Gefragt wird nicht meher, woher der einzelne kommt, sondern was er ist und was er leistet. On ne demandera plus d'où vient tel individu, mais ce qu'il est et ce qu'il réalise (leistet) Dr Goebbels ». Autant de maximes qui répètent donc au cœur des entreprises ce principe énoncé par Hitler au Congrès de Nuremberg en 1933 sur la preuve de l'appartenance raciale par la capacité de réalisation.

Puis viennent deux pages d'exemples de « sentences murales », composée chacune dans un caractère typographique différent mais dont la forme, réputée authentiquement "germanique ", est évidemment essentielle ; comme l'écrit en effet un idéologue nazi, «le caractère allemand (...) est pour nous l'expression de notre être propre et particulier, de notre être allemand même, que les mots ne suffisent pas à décrire ${ }^{25}$ ». Si certains de ces Wandsprüche citent Hitler, Hindenburg ou le chef du Front du Travail Robert Ley, la plupart provient de Grands Allemands d'un passé plus ou moins récent : militaires et politiques, tels Bismarck ou Moltke, mais surtout philosophes et penseurs allemands comme Nietzsche, Kant, Arndt, Lagarde, Schiller ou Fichte. Mais tous doivent "contraindre " chaque travailleur à faire retour sur soi-même, c'est-à-dire à communier avec la substance passée et présente de sa race. 
Fig.1

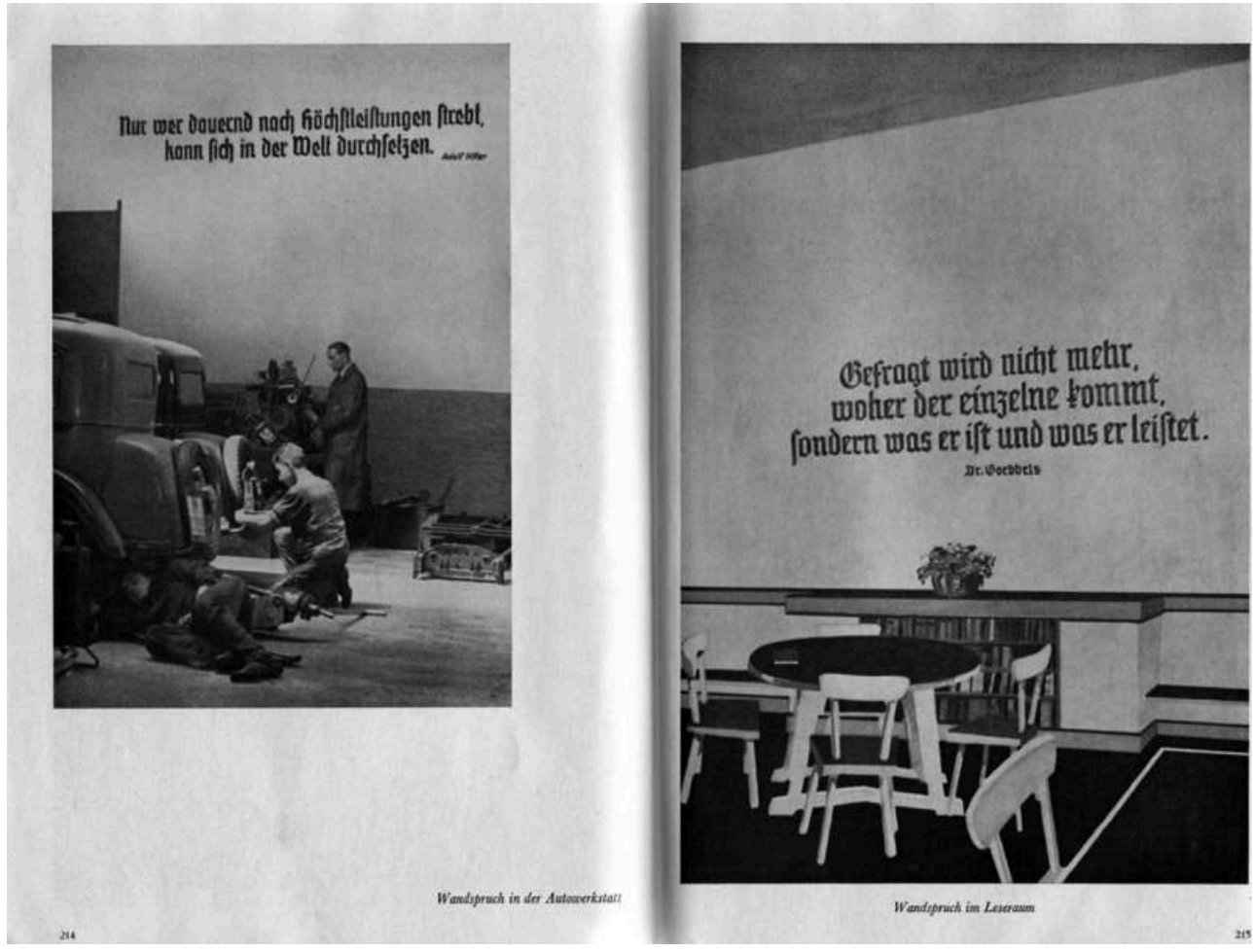

« Sentences murales » ou Wandsprüche dans les entreprises allemandes ("Taschenbuch Schönheit der Arbeit", Berlin, 1938)

Fig.2

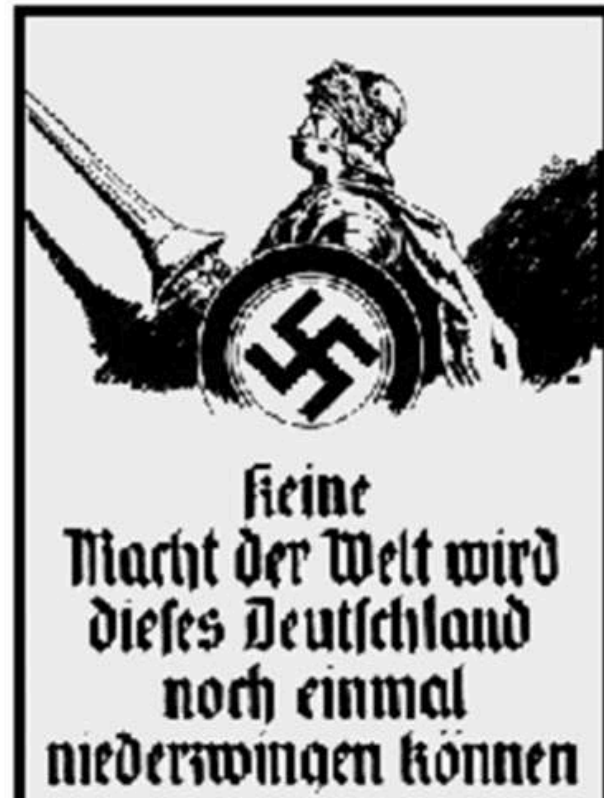

Anotf firler

"Sentence hebdomadaire » ou Wochenspruch : « II n'est aucune puissance au monde que cette Allemagne ne puisse à nouveau soumettre. Adolf Hitler ». 
A ces Wandsprüche qui s'étalent dans toutes les entreprises répondent, plus nombreux encore, les Wochensprüche, les "sentences hebdomadaires » qui se répandent un peu partout par voie d'affiches et dont tout Allemand est par conséquent le destinataire potentiel. Beaucoup de ces affichettes sont illustrées, comme cette image moyenâgeuse se combinant avec une sentence de Hitler, s'inscrivant en lettres nécessairement gothiques (Fig. 2) : «Keine Macht der Welt wird dieses Deutschland noch einmal niederzwingen können - Il n'est aucune puissance au monde que cette Allemagne ne puisse à nouveau soumettre ». Un héroïque chevalier teutonique voit son bouclier s'actualiser de la croix gammée du parti, comme pour mieux affirmer que l'Allemagne d'aujourd'hui hérite de sa race non seulement la même force, mais aussi les mêmes ennemis contre lesquels se poursuit le même combat.

Fig.3

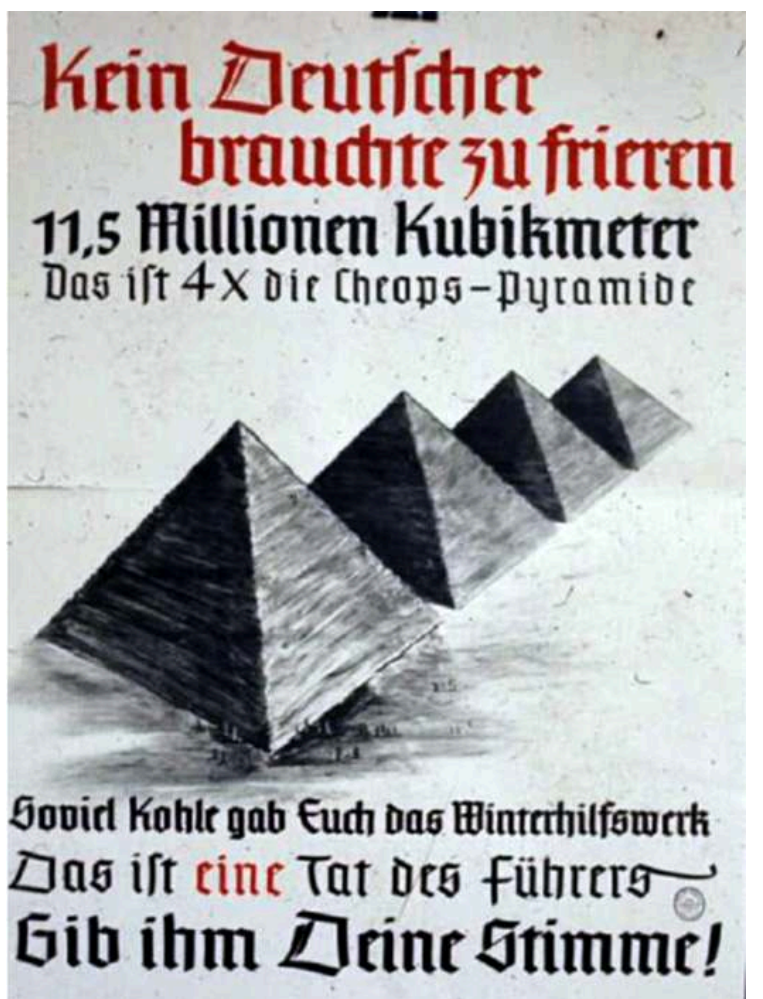

«Sentence hebdomadaire » ou Wochenspruch: «Aucun Allemand n'aura plus froid. 11, 5 millions de mètres cubes. C'est 4 fois la pyramide de Cheops. Voilà tout le charbon que vous donne le Secours d'hiver. C'est un acte du Führer. Donne-lui ta voix!».

Plus étonnant, un autre Wochenspruch cherche à convaincre les Allemands de voter pour Hitler parce qu'il est le Führer capable de surpasser les exploits des anciens Egyptiens pour protéger son peuple (Fig.3) : «Aucun Allemand n'aura plus froid. 11,5 millions de mètres cubes. C'est 4 fois la pyramide de Cheops. Voilà tout le charbon que vous donne le Secours d'hiver. C'est un acte du Führer. Donne-lui ta voix!» Aussi étrange que cela paraisse, cette référence aux pyramides de l'Egypte ancienne n'a rien de fortuit : tout comme aux Grecs et aux Romains de l'Antiquité, les nazis s'identifient aussi à ces autres "Aryens" qu'étaient, selon eux, les anciens Egyptiens dont ils revendiquent également l'héritage. Ainsi, lorsqu'au Congrès de Nuremberg de 1935 
Hitler justifie les grands programmes architecturaux qu'il projette pour l'Allemagne nouvelle, il déclare avec l'emphase qui lui est habituelle :

«Que seraient les Egyptiens sans leurs pyramides et leurs temples, (...) les Grecs sans Athènes et sans l'Acropole, Rome sans ses monuments, nos générations d'empereurs germains sans les cathédrales (...)? Aucun peuple ne vit plus longtemps que les documents de sa culture! (Kein Volk lebt länger als die Dokumente seiner Kultur) ${ }^{26}$ ».

Mais ces grands exemples monumentaux du passé «aryen », à l'aune desquels Hitler cherchait sans cesse à mesurer sa propre importance et sa propre "grandeur » historique, il ne lui fallait pas seulement les égaler, il lui fallait bien davantage encore les surpasser. Lui qui, dans Mein Kampf, avait défini la Kultur que le nazisme cherchait à promouvoir comme étant "fondée sur l'esprit grec et la technique allemande ${ }^{27}$ ", il reste profondément convaincu que cette technique prolongera la vitalité de son peuple par des constructions plus vastes et plus durables, capables d'abriter plus longtemps le génie protecteur de sa race. Aussi dérisoire soit-il, ce Wochenspruch citant visuellement la pyramide chéops en affirmant, pour inviter à voter en faveur de Hitler, que la technique allemande est capable d'en dépasser quatre fois le volume en charbon pour protéger les Allemands, ce Wochenspruch entre donc exactement dans la perspective qui est celle du Führer: surpasser les exempla du passé, mais toujours les citer pour apporter la preuve tangible de leur surpassement par les Leistungen, par les performances de la race dans son actualité présente.

Fig.4

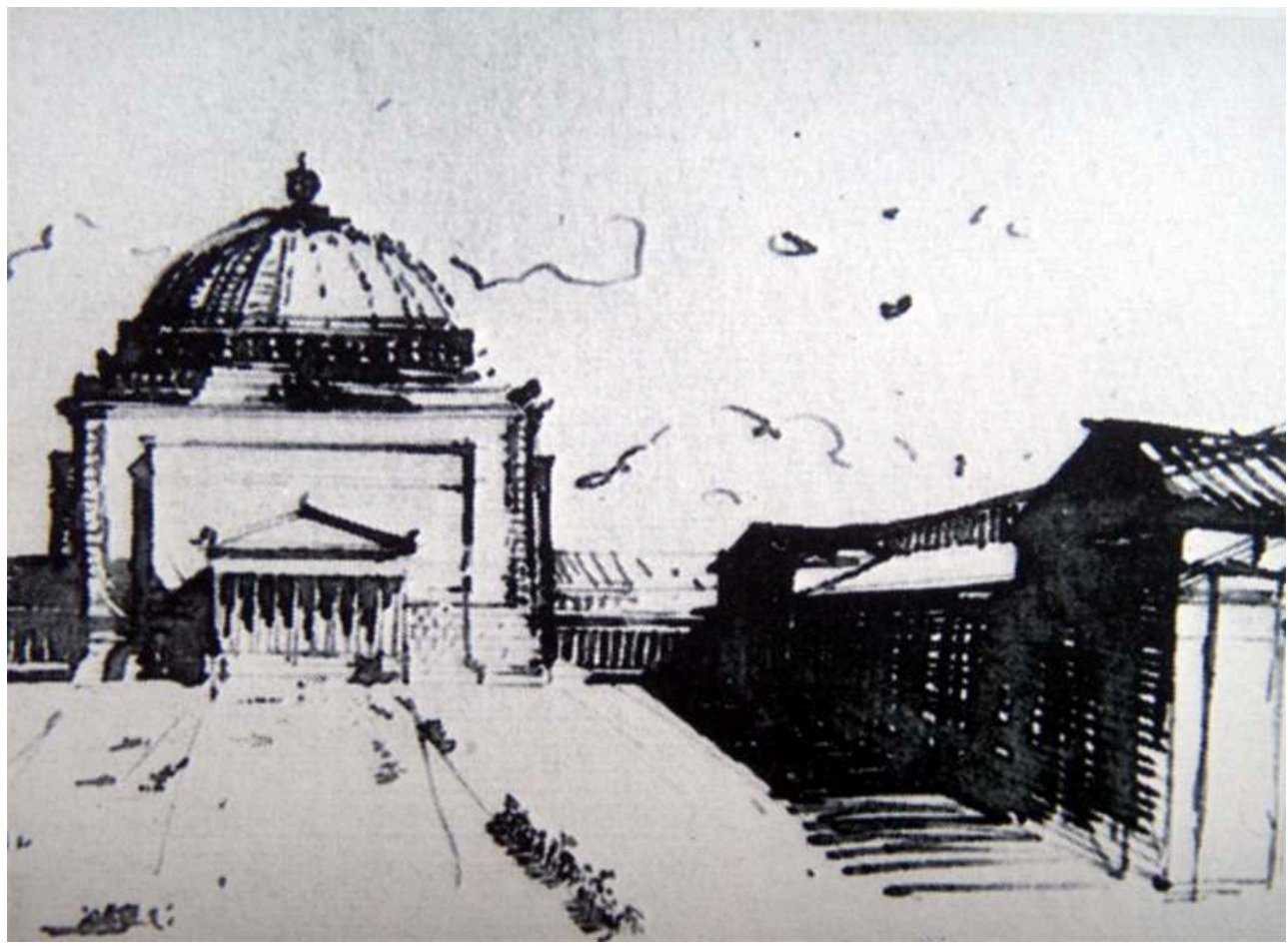

Esquisse d'Adolf Hitler pour le Grand Dôme de la future Germania (Berlin), capitale du Grand Reich, 1925. 
Fig.5

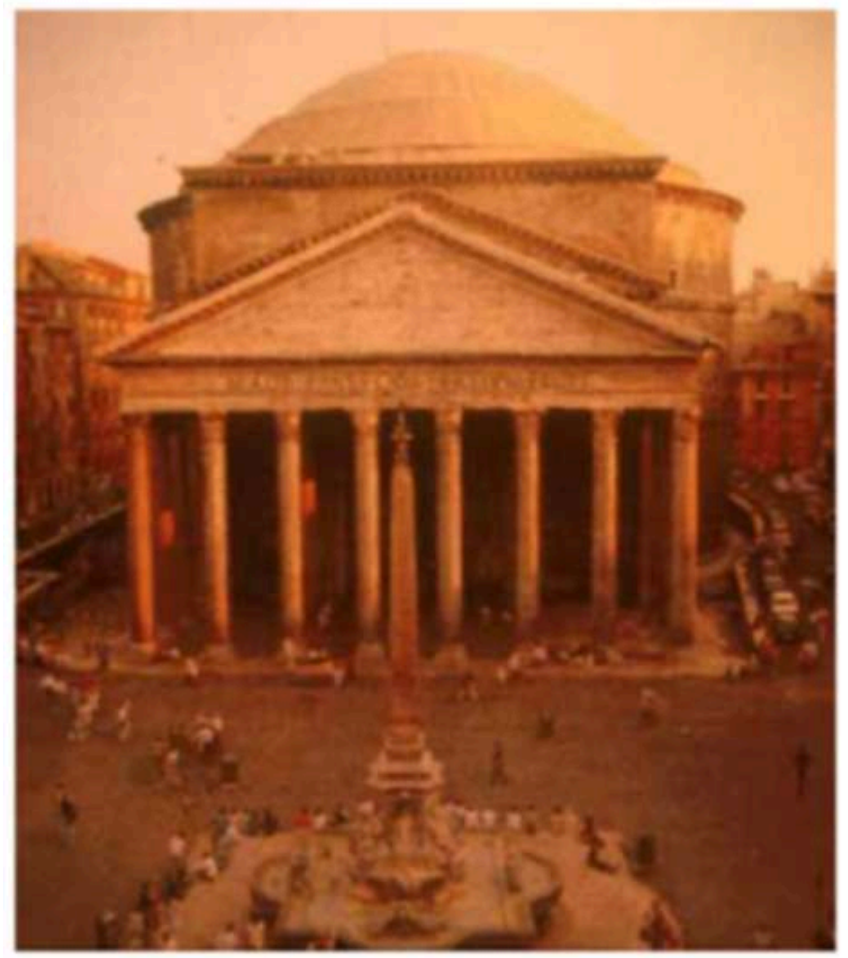

Panthéon d'Hadrien, Rome (ca. 125).

Fig.6

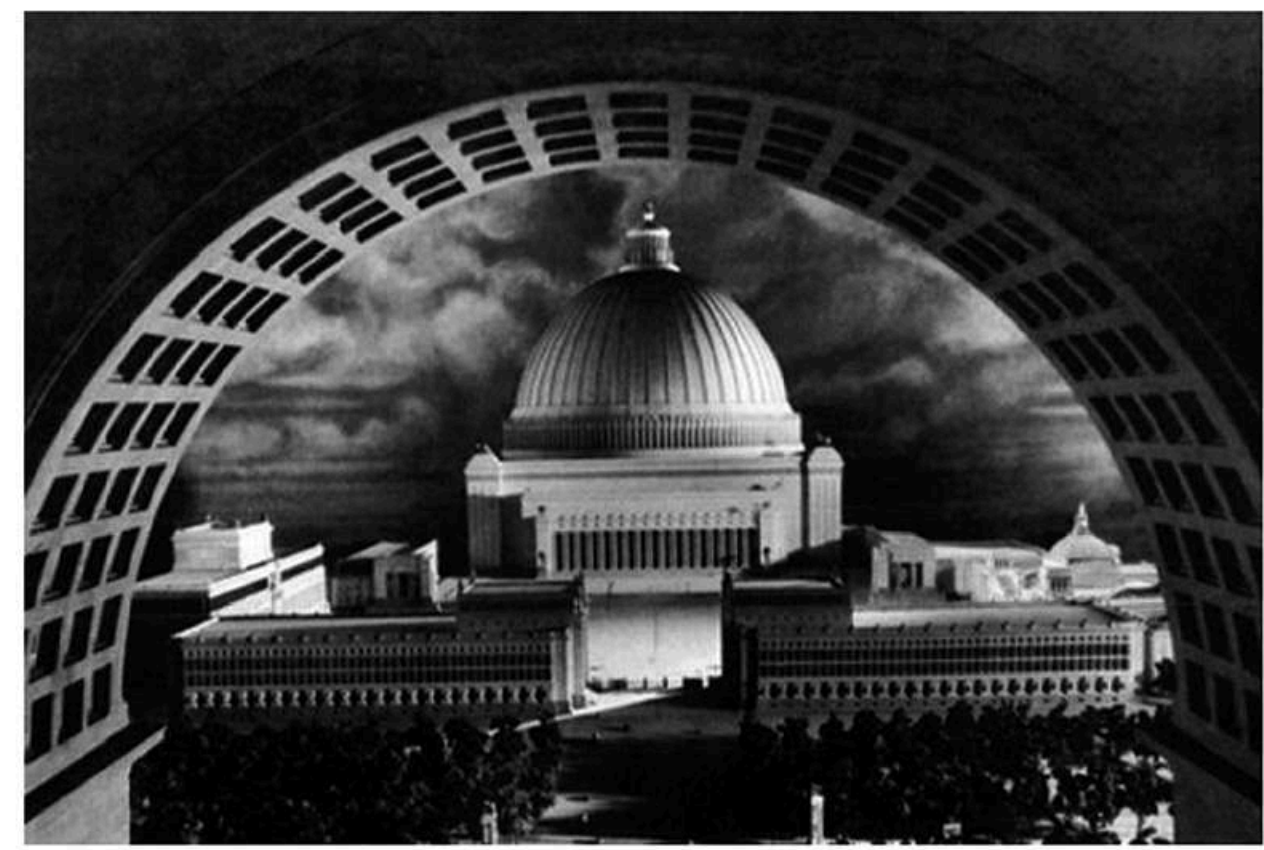

Maquette d'Albert Speer pour le Grand Dôme de la future Germania (1938)

Ce principe n'est nulle part mieux affirmé ni plus visible que dans le domaine de l'architecture sur lequel Hitler règne sans partage, avec la complicité de l'architecte 
Albert Speer ${ }^{28}$. Lorsque ce dernier entreprend de réaliser le nouveau Berlin, la capitale de l'Empire éternel qui devra porter le nom de "Germania », il obéit d'abord au Führerprinzip en citant très fidèlement une esquisse de Hitler dessinée en 1925 (Fig.4) pour le Grand Dôme, ou Volkshalle qui devrait abriter les représentants d'un empire de 140 millions d'hommes. A l'évidence, l'esquisse de Hitler était elle-même une citation du Panthéon que l'empereur Hadrien avait fait édifier à Rome (Fig. 5), mais d'un Panthéon très largement surdimensionné. Or la maquette du Grand Dôme (Fig. 6) que Speer offre à Hitler pour son anniversaire, le 20 avril 1937, se présente donc non seulement comme une citation de Hitler citant le Panthéon, mais elle cite encore d'autres éléments de l'architecture du Panthéon dont Hitler ne s'était pas préoccupé et dont elle surpasse encore les dimensions. Ainsi en va-t-il par exemple de l'ouverture circulaire ménagée dans la coupole, elle-même structurée par des caissons identiques à ceux de l'édifice romain. De cette ouverture, Speer rappellera plus tard lui-même qu'avec ses 46 mètres de diamètre, elle dépassait non seulement le diamètre de " toute la coupole du Panthéon ", qui n'est que de 43 mètres, mais aussi les 44 mètres du diamètre de la coupole de Saint-Pierre de Rome. Quant au volume intérieur, notera Speer avec une certaine émotion, il «faisait dix-sept fois celui de la basilique SaintPierre $^{29} »$. Enfin, la lanterne surmontant la coupole du Grand Dôme n'est elle non plus rien d'autre qu'une citation très amplifiée de la lanterne de Saint-Pierre de Rome - à ceci près que, sur l'ordre express donné par Hitler au début de l'été $1939^{30}$, l'aigle du Reich tenant dans ses serres le globe terrestre s'y substitue à la croix du christianisme.

Fig.7

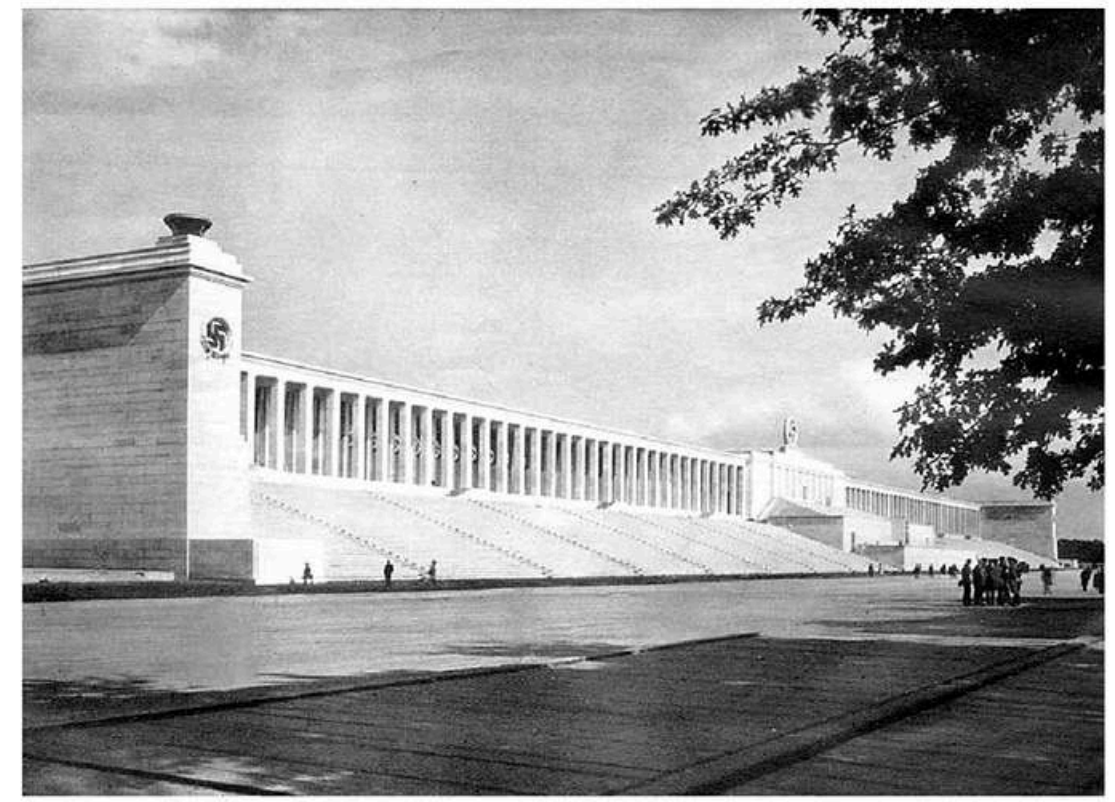

Die Zeppelintribüne nach der Fertigstellung im Jahre 1937; n. BOCK (1938).

Albert Speer, Tribune de l'Esplanade Zeppelin, Nuremberg, 1937. 


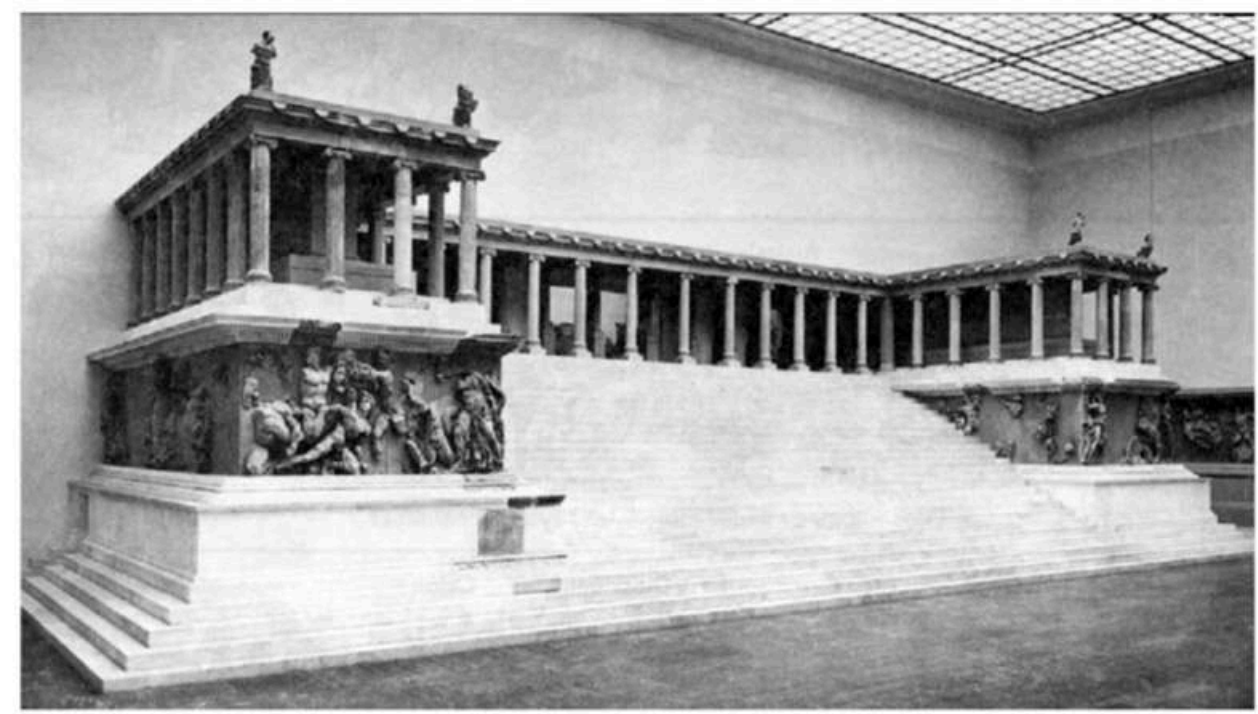

Autel de Pergame (180-159 av.JC), reconstruit à la fin du XIXe siècle dans le Pergamon Museum de Berlin qui fut édifié à cette fin.

Parmi les grands travaux menés par Speer sous la haute autorité de Hitler, le Grand Stade de Nuremberg (maquette) se présente ouvertement comme citation du Stade Panathenikon d'Athènes qui cependant, loin d'être "antique » et "authentique », avait été construit pour les premiers Jeux du renouveau olympique, en 1896. Quant à la Tribune Zeppelin (Fig.7), construite en 1937 sur l'esplanade du même nom, elle est non moins explicitement une citation, quelque peu adaptée il est vrai, de l'autel de Pergame $^{31}$ (Fig.8), tout comme la Kongresshalle de l'architecte Ludwig Ruff, laquelle ne sera jamais édifiée, est une citation, très adaptée cette fois, du Colisée de Rome. On peut enfin mentionner, parmi tant d'autres exemples, la Marmorgalerie édifiée par Speer en 1938 pour la nouvelle chancellerie du Reich : avec ses 146 mètres de long, elle double délibérement la Galerie des Glaces de Versailles dont elle est aussi l'évidente citation. «Vous verrez, exulte Hitler, ils prendront conscience de la puissance et de la grandeur du Reich allemand, quand ils auront fait tout ce chemin depuis l'entrée jusqu'à la salle de réception ${ }^{32}$ !»

«C'est pour accroître notre autorité que s'élèvent nos édifices » déclare le Führer en 1937. Il n'en faut pas douter : c'est aussi pour accroître cette autorité qu'il fait pratiquer l'inclusion par citation de l'auctoritas d'un édifice ancien dans son amplification démesurée. Toujours, il conçoit et ordonne la réalisation des plus grandes tribunes, des plus hautes coupoles, des plus gigantesques arcs de triomphe du monde et « de tous les temps ${ }^{33}$ ». Elias Canetti l'avait remarqué : "Chacune de ses entreprises, mais aussi ses souhaits les plus profonds, sont dictés par la contrainte de surpasser: on pourrait même le qualifier d'esclave du surpassement ${ }^{34} »$. Surdimensionnée, l'architecture des grands édifices publics que Hitler projette avec Speer lui semble pouvoir dilater à l'infini le présent et contenir ainsi toute la "substance du peuple ", passée et à venir.

Tout comme les citations verbales, les citations architecturales des grands monuments, provenant d'un passé supposé commun aux Aryens dont le peuple allemand serait l'héritier le plus authentique, fonctionnent donc comme autant d'auctoritates, issues de la race pour exercer leur puissance sur la race. Mais à la différence des citations 
verbales, leur surdimensionnement devrait produire une surautorité, car seule la citation architecturale est capable de transformer chacun des membres de la Volksgemeinschaft en témoin de la grandeur perpétuée de la race. Ne se donnant pas seulement à lire, à voir ou à entendre, l'espace architectural devient, par son caractère enveloppant et "haptique ", l'espace d'un Erlebnis, d'une "expérience vécue » et participative, s'emparant de chacun pour l'intégrer au mythe de la race dont ces «réalisations culturelles» prouvent la supériorité. A la différence enfin des innombrables citations peintes et sculptées destinées à fournir des modèles à la Communauté du Peuple, la citation architecturale, procédant par immersion, plonge ceux qu'elle absorbe au cœur du mythe réalisé, les métamorphosant instantanément en «Grecs » ou en « Romains» modernes - abolissant l'histoire par la fabrication d'une mémoire du présent. Elle suscite le sentiment que l'expérience actuelle a déjà été "vécue ", cette illusion de fausse reconnaissance que Bergson analysait plus justement comme «le souvenir du présent». Parce que le présent, disait-il, se scinde nécessairement en même temps qu'il se pose, parce que la formation du souvenir est contemporaine à la perception actuelle, celui qui éprouve ce sentiment « devient plus ou moins étranger à lui-même et comme 'automatisé" ". Le caractère pour lui « inévitable » et prévisible de ce tout qui se fait et se dit alors, la conviction qu'il reconnaîtra aussitôt chacun des moments qui sont encore à venir font de lui un acteur jouant " automatiquement son rôle, s'écoutant et se regardant jouer $»^{35}$. S'il est vrai, comme l'écrivait Jünger en 1932, que le « bourgeois » donne puissance sur lui-même «à toute grandeur historique qu'il est en train de considérer ", et si pour cette raison même "on peut le battre à l'aide de n'importe quelle citation", alors la citation architecturale est bien cet espace contraignant le «bourgeois » à jouer et ré-citer un rôle qu'il n'a pourtant jamais appris.

Un dernier type de citation illustre clairement ce principe: la "citation» des «Martyrs » durant la cérémonie du 9 novembre, qui commémore la mémoire des seize nazis morts lors du putsch manqué de 1923 à Munich - une cérémonie chaque année retransmise en direct dans l'Allemagne entière par les postes de radio publics. A l'appel du nom de chacun de ces fameux Blutzeugen - de ces «martyrs » ou, plus littéralement, de ces « témoins du sang »-, le chœur des Hitler Jugend répond par le mot : « présent ! ». Et la définitive rédemption des vaincus d'hier, tombés lors du putsch manqué, s'effectue au moment précis du rituel où est prononcée cette parole : «Und Ihr habt doch gesiegt ! Et vous avez tout de même vaincu !»- transformant les vaincus en vainqueurs. Par là se révèle d'un coup, à travers ce rituel emprunté aux fascistes italiens, la nature de la relation que le national-socialisme prétend établir avec un passé mythifié : faire affleurer à la surface du présent toute la mémoire raciale, toute cette mémoire du sang qu'il s'est efforcé de construire, pièce par pièce, à coup de citations, pour fabriquer le Reich éternel ; c'est-à-dire faire jouer aux vivants le rôle des morts. Comme le dit alors Baldur von Schirach, le chef des Jeunesses hitlériennes : «Il n'y a rien de plus vivant en Allemagne que nos morts $»^{36}$.

31 L'immense travail de réalisation ou de Leistung qui emporte un peuple vers son Troisième Reich idéal est assurément tout le contraire d'un travail du deuil : c'est un travail d'anamnèse fondé sur la «foi » dans son propre pouvoir de redonner un corps et une vie à l'objet perdu. La citation aura donc été érigée par le nazisme tout à la fois en programme et en moyen de gouvernement par la persuation contraignante. On peut la nommer propagande, à la condition toutefois de ne jamais entendre par là le camouflage d'une réalité sordide, mais bien plutôt la construction de cette réalité sous 
la forme du mythe. Josef Peter Stern avait clairement perçu cette mytho-poḯtique du nazisme, lorsqu'il comparait sa propagande à " une structure poétique rudimentaire »: «Ôtez la 'propagande' de ce qu'elle vise à promouvoir, ce qui subsiste n'est pas un programme politique privé de ses moyens de réalisation, mais un contenu que l'absence de contenant rend défectueux : un pur néant ${ }^{37}$.

C'est dans ce sens que le régime nazi fait de la citation le principe du gouvernement raciste-völkisch : comme auctoritas s'opposant à tout dialogue et à tout débat, excluant par principe tout ce qui ne lui est pas artgleich, la citation est plus qu'un ordre donné depuis les profondeurs de l'âme aryenne et racialement ou "généalogiquement" transmise, elle est plus qu'une injonction se communicant au Volkskörper entier : elle est l'écho de la race se propageant - performativité pure. Mieux encore: par la dimension proprement religieuse qui lui est conférée, la citation est au corps éternel de la race ce que l'hostie est au corps du Christ : c'est par l'absorbtion et la consommation de toutes ces citations verbales et plastiques que chacun, témoignant de son appartenance au nouveau corps mystique, donne vie et consistance à la Communauté de race.

Assurément, citation et Leistung, comprise comme "réalisation » ou "performance ", entretiennent sous le national-socialisme un lien de nature singulière. A la longue en effet, tout ce qui relève de la production et de la reproduction du même - depuis la simple imitation artistique jusqu'à la reproduction photographique d'un paysage "allemand", depuis la production industrielle d'objets manufacturés "allemands " jusqu'à la reproduction biologique de la race - acquiert sous le Troisième Reich le statut de citation, entendue comme autocitation, c'est-à-dire incluant et amplifiant les Leistungen ou réalisations précédentes de la "race créatrice de Kultur ». Et si, comme le dit Hitler, «on ne peut pas conclure de la race à la capacité, mais de la capacité à la race », cela signifie non seulement que par la citation se vérifie la filiation légitime, l'autocitation devenant preuve de l'appartenance à la race, mais aussi qu'elle est la seule authentique « réalisation » de l'essence éternelle du Volk. Le nazisme s'autodéfinit ainsi comme un corps autopoḯtique dont l'autoproduction n'a d'autre fin que sa propre extension, que la croissance virtuellement infinie de lui-même.

\section{NOTES}

1. Walter Benjamin, «Sur le concept d'histoire» (1940), CEuvres, trad. M. de Gandillac, R. Rochlitz et P. Rusch, Paris, Gallimard, 2000, vol. 3, p. 432-433.

2. Id., ibid., p. 429.

3. Martin Heidegger, Etre et temps (Sein und Zeit, 1927), trad. E. Martineau, Paris, Authentica, 1985 , § 73, p. 385-386.

4. Ernst Jünger, Le Travailleur (Der Arbeiter, 1932), trad. J. Hervier, Paris, Christian Bourgois, 1989, p. 259 (je souligne).

5. Id., ibid., p. 261.

6. Id., ibid., p. 266-270.

7. Id., ibid., p. 259 (je souligne). 
8. Adolf Hitler, Mein Kampf, Munich, Franz Eher, 1940 (1925 et 1927 pour la 1e éd.), p. 598 ; Mon Combat, trad. J. Gaudefroy-Demombynes et A. Calmettes, Paris, Nouvelles Editions Latines, 1934, p. 531.

9. Sur les rapports de Chateaubriand à l'Historia magistra, voir François Hartog, Régimes d'historicité. Présentisme et expériences du temps, Paris, Le Seuil, 2003, ch. 3.

10. Mon Combat, p. 152, Mein Kampf, p. 166 : «Und setzet ihr nicht das Leben ein, / Nie wird euch das Leben gewonnen sein - Et si vous n'engagez pas votre vie elle-même, / Jamais vous ne la gagnerez, votre vie».

11. Mon Combat, p. 230 : « (Les Juifs sont) les grands maîtres du mensonge », qui fait pendant à la citation d'un officier anglais affirmant que "Sur trois Allemands, il y a un traître »; Mein Kampf, p. 253.

12. Mon Combat, p. 480 ; Mein Kampf, p. 540.

13. Mon Combat, p. 487 ; Mein Kampf, p. 548.

14. Mon Combat, p. 235 : «Le chapeau à la main, on peut traverser tout le pays »; et p. 656 : «Le désir est père de l'idée »; Mein Kampf, p. 258 et 746.

15. Carl Schmitt, Staat, Bewegung, Volk. Die Dreigliederung der politischen Einheit, Hamburg, Hanseatische Verlaganstalt, 1933, p. 42 («IV. Führertum und Artgleichheit als Grundbegriffe des nationalsozialistischen Rechts »).

16. Id., ibid.

17. Mon Combat, p. 382 ; Mein Kampf, p. 422-423.

18. Hannah Arendt, «Le concept d'histoire » (trad. Patrick Lévy), La crise de la culture. Huit exercices de pensée politique, Paris, Gallimard, 1972, p. 68.

19. Mon Combat, p. 338 ; Mein Kampf, p. 372.

20. Alfred Rosenberg, Der Mythus des 20. Jahrhunderts, Munich, Hoheneichen-Verlag, 1941, p. 514-515.

21. Id. ibid, p. 303.

22. Paul Schultze-Naumburg, Kunst und Rasse, Munich, Lehmanns, 1928, p. $74-77$ (c'est lui qui souligne).

23. Adolf Hitler, Die Reden Hitlers am Reichsparteitag 1933, Munich, Franz Eher, 1934, p. 37.

24. Anatol von Hübbenet, Das Taschenbuch Schönheit der Arbeit, Berlin, Verlag der deutschen Arbeitsfront, 1938, p. 212.

25. R. Koch, «Die deutsche Schrift », Die Neue Literatur, 1937, p. 538, reproduit in Joseph Wulf, Litteratur und Dichtung im Dritten Reich. Eine Dokumentation, Frankfurt/Main, Berlin, Wien, Ullstein, 1983, p. 380 (je souligne).

26. «Rede Hitlers auf der Kulturtagung des Reichsparteitages in Nürnberg 1935 », reproduit par B. Hinz, Die Malerei im deutschen Faschismus. Kunst und Konterrevolution, Munich, Karl Hanser, 1974, p. 139-152 (ici p. 142-143).

27. Mon Combat, p. 289 ; Mein Kampf, p. 318.

28. Voir Alex Scobie, Hitler's State Architecture. The Impact of Classical Antiquity, The Pennsylvania State University Press, University Park and London, 1990.

29. Albert Speer, Au cœur du Troisième Reich, trad. Michel Brottier, Paris, Fayard, 1971, p. 218.

30. Id., ibid., p. 229.

31. Speer souligne lui-même que « l'influence de l'autel de Pergame était évidente », ibid., p. 80.

32. Id., ibid., p. 148. Voir aussi Angela Schönberger, Die Neue Reichskanzlei von Albert Speer. Zum Zusammenhang von nationalsozialistisscher Ideologie zur Architektur, Berlin, Gebr. Mann, 1981, p. 94-95.

33. Voir Joachim Fest, Hitler, trad. Guy Fritsch-Estrangin, Paris, Gallimard, 1973, vol. II (Le Führer), p. 182-184. 
34. Elias Canetti, «Hitler, d'après Speer », La Conscience des mots, trad. R. Lewinter, Paris, Albin Michel, 1984, p. 209. « Mais en cela, ajoute Canetti, il n'est pas le seul. Si l'on devait qualifier d'un trait l'essence de notre société, on ne pourrait trouver que ceci : la contrainte de surpasser. »

35. Henri Bergson, «Le souvenir du présent et la fausse reconnaissance » (1908), L'énergie spirituelle (1919), in CEuvres, Edition du centenaire, Paris, Presses universitaires de France, 1963, p. 897-930.

36. Cité par G. Kaufmann, Das kommende Deutschland. Die Erziehung der Jugend im Reich Adolf Hitlers, Berlin, 1943 (3e édition), p. 301, reproduit par Hans-Jochen Gamm, Führung und Verführung. Pädagogik des National-sozialismus, Munich, Paul List, 1990, p. 358.

37. Josef Peter Stern, Hitler, le Führer et le peuple (1975), trad. Suzanne Lorme, Préface Pierre Ayçoberry, Paris, Flammarion, 1985, p. 109.

\section{INDEX}

Mots-clés : citation nazie, monument, propagande, sentence murale

\section{AUTEUR}

\section{ERIC MICHAUD}

Directeur d'études à l'EHESS. Il a notamment publié Théâtre au Bauhaus (L'Age d'Homme, 1978), Hypnoses (avec M. Borch-Jacobsen et J.-L. Nancy, Galilée, 1984), La fin du salut par l'image (Jacqueline Chambon, 1992), Un art de l'éternité. L'image et le temps du national-socialisme (Gallimard, 1996), Fabriques de l'homme nouveau, de Léger à Mondrian (Carré, 1997), Histoire de l'art : une discipline à ses frontières (Hazan, 2005). Il prépare actuellement un ouvrage sur le nationalisme et le racisme dans l'historiographie de l'art.

Eric.Michaud@ehess.fr 